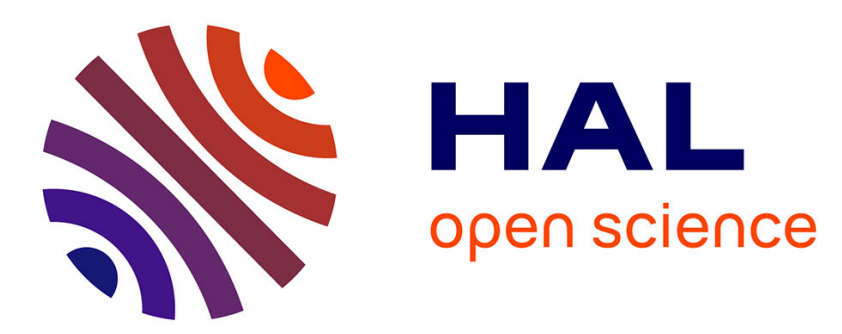

\title{
Screening Methodology for the Efficient Pairing of Ionic Liquids and Carbonaceous Electrodes Applied to Electric Energy Storage
}

Julio F. Jover, Rafael Lugo, Hervé Toulhoat, Patrice Simon, Theodorus de Bruin

\section{To cite this version:}

Julio F. Jover, Rafael Lugo, Hervé Toulhoat, Patrice Simon, Theodorus de Bruin. Screening Methodology for the Efficient Pairing of Ionic Liquids and Carbonaceous Electrodes Applied to Electric Energy Storage. Journal of Physical Chemistry C, 2014, vol. 118 ( $\left.\mathrm{n}^{\circ} 2\right)$, pp. 864-872. 10.1021/jp409995q . hal-01153082

\section{HAL Id: hal-01153082 \\ https://hal.science/hal-01153082}

Submitted on 19 May 2015

HAL is a multi-disciplinary open access archive for the deposit and dissemination of scientific research documents, whether they are published or not. The documents may come from teaching and research institutions in France or abroad, or from public or private research centers.
L'archive ouverte pluridisciplinaire HAL, est destinée au dépôt et à la diffusion de documents scientifiques de niveau recherche, publiés ou non, émanant des établissements d'enseignement et de recherche français ou étrangers, des laboratoires publics ou privés. 


\section{Open Archive TOULOUSE Archive Ouverte (OATAO)}

OATAO is an open access repository that collects the work of Toulouse researchers and makes it freely available over the web where possible.

This is an author-deposited version published in : http://oatao.univ-toulouse.fr/ Eprints ID : 11348

To link to this article : DOI:10.1021/jp409995q

URL : http://dx.doi.org/10.1021/jp409995q

\section{To cite this version :}

Jover, Julio F. and Lugo, Rafael and Toulhoat, Hervé and Simon, Patrice and De Bruin, Theodorus Screening Methodology for the Efficient Pairing of Ionic Liquids and Carbonaceous Electrodes Applied to Electric Energy Storage. (2014) Journal of Physical Chemistry C, vol. 118 (n ${ }^{\circ}$ 2). pp. 864-872. ISSN 1932-7447

Any correspondance concerning this service should be sent to the repository administrator: staff-oatao@ listes-diff.inp-toulouse.fr 


\title{
Screening Methodology for the Efficient Pairing of lonic Liquids and Carbonaceous Electrodes Applied to Electric Energy Storage
}

\author{
Julio F. Jover, ${ }^{\dagger}$ Rafael Lugo, ${ }^{\dagger}$ Hervé Toulhoat, ${ }^{\dagger}$ Patrice Simon, ${ }^{\ddagger}, \S$ and Theodorus de Bruin ${ }^{* \dagger}$ \\ ${ }^{\dagger}$ IFP Energies nouvelles, 1 et 4 avenue de Bois-Préau, 92852 Rueil-Malmaison, France \\ †Université Paul Sabatier de Toulouse, CIRIMAT, UMR-CNRS 5085, 31062 Toulouse Cedex 4, France \\ ${ }^{\S}$ Réseau national sur le Stockage Electrochimique de l'Energie, FR CNRS 3459, 80039 Amiens Cedex, France
}

\begin{abstract}
A model is presented that correlates the measured electric capacitance with the energy that comprises the desolvation, dissociation and adsorption energy of an ionic liquid into carbonaceous electrode (represented by single-wall carbon nanotubes). An original methodology is presented that allows for the calculation of the adsorption energy of ions in a host system that does not necessarily compensate the total charge of the adsorbed ions, leaving an overall net charge. To obtain overall negative (favorable) energies, adsorption energies need to overcome the energy cost for desolvation of the ion pair and its dissociation into individual ions. Smaller ions, such as $\mathrm{BF}_{4}{ }^{-}$, generally show larger dissociation energies than anions such as $\mathrm{PF}_{6}{ }^{-}$or $\mathrm{TFSI}^{-}$. Adsorption energies gradually increase with decreasing pore size of the CNT and show a maximum when the pore size is slightly greater than the dimensions of the adsorbed ion and the attractive van der Waals forces dominate the interaction. At smaller pore diameters, the adsorption energy sharply declines and becomes

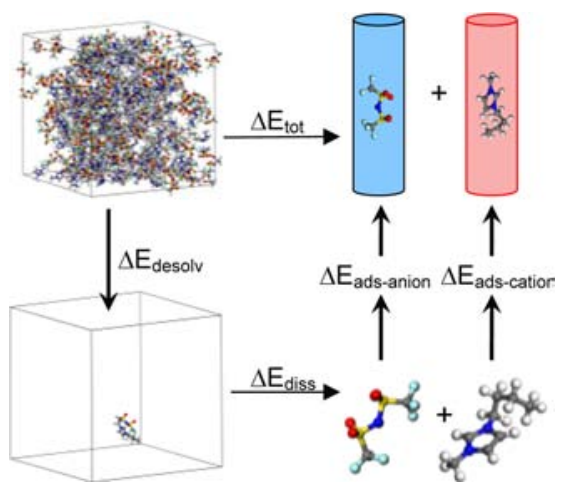
repulsive as a result of geometry deformations of the ion. Only for those diameters where the adsorption reaches maximum values is the adsorption energy sufficiently negative to balance the positive dissociation and desolvation energies. We present for each ion (and ionic liquid) what the most adequate electrode pore size should be for maximum capacitance.
\end{abstract}

\section{INTRODUCTION}

For the migration from fossil-fuel/coal energy generation to be successful, one significant issue remains to be resolved: the discontinuous nature of most renewable and greener energy sources. Photovoltaic plants and windmills farms, for example, cannot compete in terms of a continuous supply of energy (or, as of today, in terms of power) with coal/gas plants or nuclear reactors. To help narrow the gap without disregarding the environmental aspects, industrial-grade ecofriendly energy storage is required. An issue facing similar difficulties but on a smaller scale is energy storage for electric vehicles, which require high energy and power density storage.

There are two main ways of storing electrical energy: in chemical bonds (as in batteries) and through charge separation (as in capacitors). A subset of the latter, called supercapacitors (or electrical double layer capacitors, EDLCs) are of special interest because of their high cyclability (similar to that of "normal" capacitors) and their specific-power/specific-energy ratio, which places them closer (in terms of usability) to batteries. ${ }^{1}$ Contrary to standard capacitors, where the electrodes are separated by an insulating material (dielectric), in EDLCs, an electrolyte separates the electrodes. The term EDLC comes from the two layers that contribute to the amount of energy to be stored. The first corresponds to the charge accumulation in the solid electrode, and the second corresponds to the adsorbed ions from the (liquid) electrolyte on its surface. In terms of power, EDLCs exceed commercially available batteries. However, when it comes to energy storage (or the length of time for which the capacitor can provide said power), supercapacitors still fall short. An attempt to find ways to increase the energy storage capacity of EDLCs is the main motivation behind this work.

There are different kinds of EDLCs depending on the materials used for the electrodes and electrolyte. They can be divided into three main groups: metal oxide, conducting polymer, and carbon-based capacitors. The last is a diverse group, as the there are many different materials and routes to obtain high-porosity materials, from coconut shells to carbidederived carbons (CDCs). ${ }^{2,3}$ Porous carbon materials are seen as an excellent choice for electrodes given their tunability and availability.

Recently, it has been shown that a decrease in the average pore size of carbonaceous materials used in EDLCs can generate an increase in the capacitance of the cell. ${ }^{1,4}$ This behavior is observed when the size of the pores is commensurate with that of the bare ions of the electrolyte, suggesting the loss of the solvation shell surrounding the ion. This phenomenon has been observed for different roomtemperature ionic liquids. ${ }^{7}$ Moreover, it has been shown that 
the pore accessibility is controlled by size effects and not charge saturation. ${ }^{7}$

To improve the understanding on a molecular level of this anomalous increase in capacitance, a significant number of molecular modeling studies have been conducted. For example, Huang et al. ${ }^{8}$ proposed a model to correlate the capacitance with the pore size and specific area of the carbonaceous electrode, among other properties. Very recently, Feng et al. published a mini-review/perspective with molecular insights from molecular simulations, ${ }^{9}$ in which they suggested that the capacitance of porous supercapacitors closely correlates with the nature and size of the room-temperature ionic liquid, the generic features of the pores of the electrode, and the applied potential.

Shim and $\mathrm{Kim}^{10}$ performed molecular dynamics simulations to quantitatively calculate the capacitance of the ethylmethylimidazolium/tetrafluoroborate ionic liquid as a function of the pore diameter of single-wall carbon nanotubes to model the electrode. In this study, they were able to correctly reproduce the experimentally observed trend of the increase in capacitance. However, the model underestimated the capacitance by a factor 2 , which the authors attributed to the absence of polarizability of the ionic liquid. Using a similar approach, Yang et al. ${ }^{11}$ calculated capacitances for the tetraethylammmonium/tetrafluoroborate ionic liquid

Very recently, using molecular dynamics, Merlet et al. ${ }^{12}$ demonstrated that, even at null potential, the electrode is already wetted by the ionic liquid of the electrolyte. Moreover, once an electric potential has been applied, the charging process involves the exchange of ions with the bulk electrolyte with no change of the volume of liquid inside the electrode. This exchange is accompanied by a partial decrease in coordination number of the ions made possible by the electrode. Importantly, for the confinement, no well-organized second layer (of counterions) can be formed, thus preventing overscreening effects, which contributes to the observed increased capacitance. Using this model, Merlet et al. calculated capacitances that were in good agreement with experimental data.

Here, the objective was not to reproduce capacitances quantitatively but, rather, to establish a model that allows for the screening of ionic liquids for a given nanoporous material, and vice versa, to maximize the capacitance. More precisely, in this semiquantitative approach, we calculate the energy involved in desolvating the ion and bringing it into a (carbon) nanopore, because this energy appears to be inversely related to the electric capacitance (vida infra). The presented methodology thus helps identifying the best match between ion and pore size, thereby allowing ionic liquids to be screened for a given nanopore distribution and vice versa. Although, experimentally, CDCs are often used as porous materials, in this work, we used single walled carbon nanotubes (CNTs), because of their well-defined pore size and geometry. In this study, a total of 18 different nanotubes with both armchair $(n, n)$ and zigzag $(n, 0)$ configurations were used (where $n$ if a characteristic vector that is commonly used when describing CNTs), with varying pore diameter from 4.5 to $14 \AA$. Additionally, our study focuses on 18 ionic liquids, formed by pairing six different cations and three different anions, which are presented in Table 1.
Table 1. Studied Cations and Anions ${ }^{13-17}$

Ion

Structure

Potential Diameter

Ref

EMIM $^{+}$

ethyl-

methylimidazolium<smiles>CCn1cc[n+](C)c1</smiles>

BMIM $^{+}$

n-butyl-

methylimidazolium

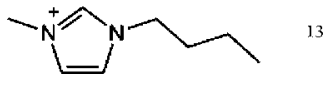

PYR $14^{+}$

n-butyl-

methylpyrrolidinium

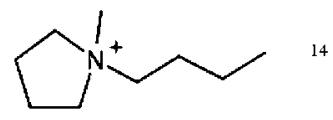

PIP $_{13}{ }^{+}$

n-propyl-

methylpiperidinium

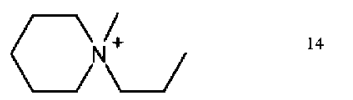

TEA $^{+}$

tetraethylammonium

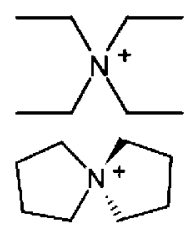

14

$\mathrm{SBP}^{+}$

spirobipyrrolidinium<smiles>F[B-](F)(F)F</smiles>

$\mathrm{BF}_{4}^{-}$

tetrafluoroborate

$\mathrm{PF}_{6}-$

hexafluorophosphate

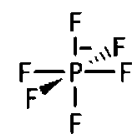

TFSI $^{-}$

bis(trifluoromethylsulfonyl)amide

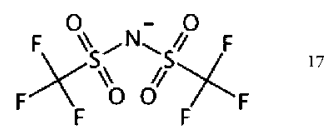

\section{METHODOLOGY}

To study the nature of the adsorption of the ionic liquid into the electrically charged electrode, a thermodynamic cycle was used as presented in Figure 1. This cycle comprises three main energy contributions that are calculated individually: (a) the energy cost to desolvate one ion pair from the bulk liquid, denoted as $E_{\text {desolv }}$ (b) the energy required to dissociate the

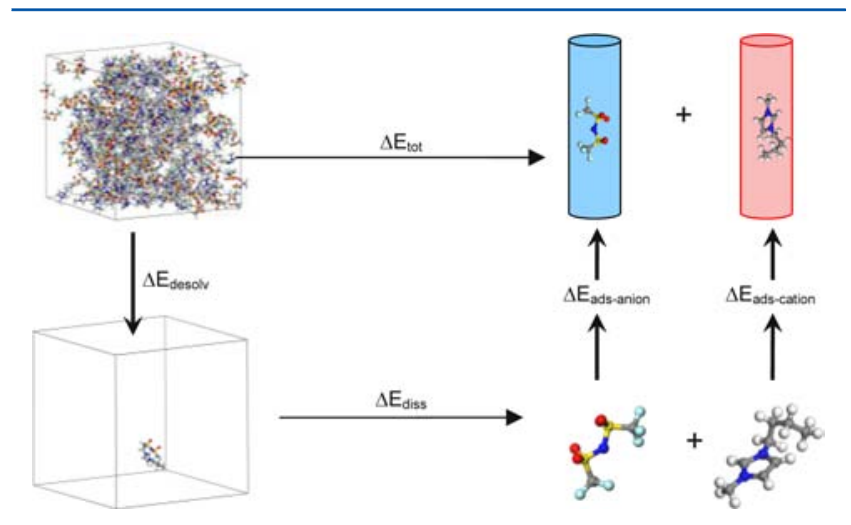

Figure 1. Thermodynamic path used to calculate the energy involved in adsorbing ions in a nanopore. 
cation from the anion, $E_{\text {diss }}$ and (c) the energy involved in the adsorption of the ions in the nanopore, $E_{\mathrm{ads}}$. By calculating these contributions independently, we could identify the limiting steps. Finally, by adding all of the terms, we recovered the overall energy of the process. In the next paragraph, we detail how each energy contribution was precisely calculated, and we end this section by giving the parameters and conditions used in each computation or simulation.

The desolvation energy $\left(E_{\text {desolv }}\right)$ was approximated by the enthalpy of vaporization and calculated as

$$
\begin{gathered}
E_{\text {desolv }} \approx \Delta H_{\text {vap }}=U_{\text {gas }}-U_{\text {liq }}+R T=\left\langle E_{\text {tot }}\right\rangle_{\text {single pair }} \\
-\frac{\left\langle E_{\text {tot }}\right\rangle_{\text {bulk }}}{n}+R T
\end{gathered}
$$

in which $U_{\text {liq }}$ and $U_{\text {gas }}$ are the average total energy of the bulk divided by the number of ion pairs (here, 250) and the average total energy of one ion pair, respectively. The total average energies of the bulk and the ion pair were obtained from NVT molecular simulations. In the case of the bulk liquid, the NVT simulations were preceded by NPT calculations to obtain the right density.

Next, the dissociation energy $\left(E_{\text {diss }}\right)$ was calculated as the energy difference between the ion pair and the individual ions. This energy resulted from DFT calculations in which only the lowest-energy conformers were taken into account. The conformers were generated by performing NVT simulations, at $298 \mathrm{~K}$, and then extracting the species at regular time intervals and relaxing their geometry.

The last step involved the adsorption of the ion in the nanopore. This is a rather delicate step, from a computational point of view: If one performs a molecular dynamics (or Monte Carlo) simulation of an ion in an unequally oppositely charged nanopore, the overall charge is not zero. To calculate the longrange electrostatic contributions, for example, using Ewald summation, this net charge needs to be compensated. However, we found that the total energy of the system becomes sizedependent if the overall charge does not equal zero. On the other hand, if we take a system with two CNTs, one tube for the cation and another for the anion, we obtain the overall adsorption energy and lose information about the contribution of each individual ion. To overcome this problem, we applied the following novel methodology presented in Figure 2. Instead of taking two pores, one per ionic species, we still used two pores $\left(\mathrm{A}\right.$ and $\left.\mathrm{A}^{\prime}\right)$, but in each pore, an ion of the same nature was inserted. However, to ensure electroneutrality, the signs of the net atomic charges of one ion were inverted. In this way, we created "virtual" ions such as $\mathrm{BF}_{4}^{+}, \mathrm{PF}_{6}^{+}$, or $\mathrm{BMIM}^{-}$. It is important to underline that only the signs of the net atomic charges were inverted and all other force field parameters, namely, bond, bond-angle, dihedral-angle, and Lennard-Jones parameters, remained unchanged. Therefore, the total energy was not impacted by this change in sign of the charges.

The overall charge of the system is now, by definition, zero, because both the studied ions and the two nanopores have opposite charges. This novel strategy presents several advantages: (1) The net charges of the nanopores can be varied independently from the number of ions inserted, as long as the two nanopores have opposite charges and the numbers of ions inserted in the two pores remain equal. (2) The number of ions in the nanopore can be varied, although the numbers in the two pores must be equal. (3) Because the average total energy calculated for the interaction between the pore and the

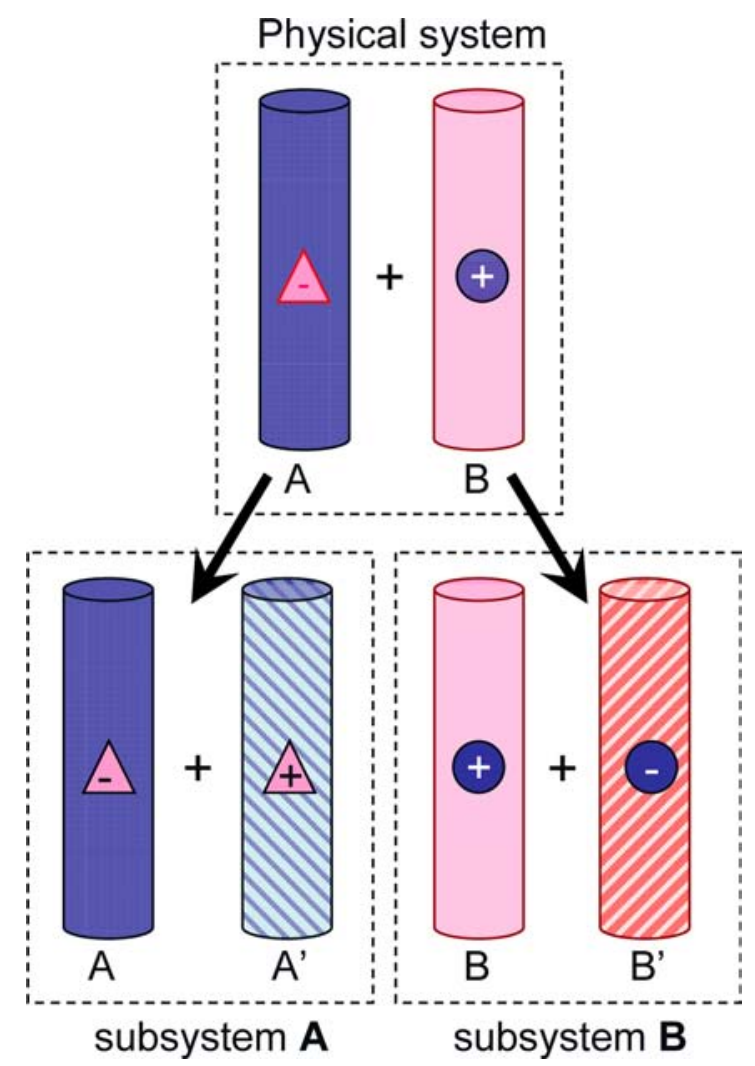

Figure 2. Top: Physical system with an anion (triangle) inserted in positively charged (purple) CNT A and a cation (circle) inserted in negatively charged (pink) CNT B. Bottom: Subsystem A presents the simulation box with CNT A and CNT A' having opposite charges with inserted an anion and positively charged "anion", respectively. Subsystem B presents the simulation box with CNT B and CNT B' having opposite charges with an inserted cation and a negatively charged "cation", respectively.

inserted ion(s) is the same for both subsystems, the statistics of the molecular dynamics is improved by a factor of 2 .

\section{COMPUTATIONAL DETAILS}

3.1. Molecular Dynamics Simulations. All molecular dynamics simulations were performed with DL_Poly Classic $(\mathrm{v} 2.18)^{18}$ with a time step of 0.5 fs. Simulations for the bulk liquid started by generating a low-density box using the PACKMOL program ${ }^{19}$ consisting of 250 ionic pairs. NPT simulations at $298.15 \mathrm{~K}$ and 1 atm typically ran for at least 5000 ps to converge the density. We applied the Nosé-Hoover thermostat and barostat constants of 0.1 and $0.1 \mathrm{ps,}$ respectively, and a van der Waals cutoff of $18.5 \AA$ was set. Ewald summation ${ }^{20}$ was used to calculate the electrostatic energy contributions, with the Ewald precision set to $1 \times 10^{-8}$. Hydrogen-carbon bonds were constrained with the SHAKE algorithm (tolerance of $1 \times 10^{-8}$ ).

The NVT simulations at $298 \mathrm{~K}$ (Nosé-Hoover thermostat, $0.1 \mathrm{fs}$ ) of a single ion pair were performed in a large cubic box (typically $10^{9} \AA^{3}$ ) to minimize interactions with periodic images. The cutoff for the van der Waals and electrostatic interactions was set to $50 \AA$, to avoid contributions from periodic images.

To calculate the adsorption energies, the setup was used as presented in Figure 2 and NVT simulations were run at $298 \mathrm{~K}$ (Nosé-Hoover thermostat, $0.1 \mathrm{fs}$ ). To minimize the 
interactions between the two CNTs and adsorbed ions, the CNTs were separated by $5000 \AA$, and the simulation box had dimensions of $10000 \times 10000 \times 51.577 \AA^{3}$. Because there is only a single ion in the nanotube, the system stabilized rather quickly, and data collection was started after 10000 steps with a production run of $1 \times 10^{6}$ steps. As DL_POLY has the ability to present energy interactions by molecular types, only the required energies, that is, the nanotube-ion interactions, were used for this study

3.2. Force Field. To be able to represent faithfully the behavior of molecules, it is necessary to have adequate forcefield parameters. In the past decade, several research groups have worked on the development and refinement of force fields for ionic liquids, including coarse-grained models, ${ }^{21,22}$ unitedatom models, ${ }^{23-25}$ and all-atom models. In the last two approaches, both polarizable and nonpolarizable atoms have been considered. Borodin and co-workers developed a polarizable-atom potential (APPLE\& $\mathrm{P}^{26-28}$ ) and performed different studies on the properties of different ionic liquids. ${ }^{29-36}$ On the other hand, there are several potentials, dved mainly from the optimized potential for liquid simulations (OPLS), among which stand out those of Sambasivarao and Acevedo ${ }^{37}$ and of Lopes and co-workers ${ }^{13,14,17}$ because of the vast numbers of ionic liquids they cover. Of these two, that of Lopes et al. has been more widely used and tested ${ }^{38}$ and was applied in this work.

CNTs were modeled as rigid bodies with fixed identical partial charges on all $\mathrm{C}$ atoms (vide infra).

3.3. Quantum Mechanical Calculations. Ion pair dissociation energies for the 17 pairs were calculated using the B3LYP functional combined with the $6-311 \mathrm{G}(\mathrm{d}, \mathrm{p})$ basis as implemented in the Jaguar suite of programs. ${ }^{39}$ The default criteria for self-consistent-field (SCF) energy convergence and geometry optimization were used.

\section{RESULTS}

4.1. Bulk Liquid: Densities and Enthalpies of Vaporization. As an initial test of our simulations, we determined the liquid-phase density. The results showed the expected behavior, with deviations of less than 5\% from the simulated and experimental values reported in the literature. ${ }^{37}$ The results are presented in the second column of Table 2 for the 18 pairs.

4.2. Enthalpy of Vaporization. Because we are interested in the relative values between different ionic liquids and not in the absolute values, we use the enthalpy to describe the desolvation energy of an ion pair. The values are reported in Table 1. It appears difficult to observe a general trend between the enthalpy of vaporization and the nature of the cation and/ or anion. Among the ionic liquids calculated, $\mathrm{BMIM} / \mathrm{BF}_{4}$, EMIM/TFSI and $\mathrm{PIP}_{13} / \mathrm{PF}_{6}$ have the smallest enthalpies of vaporization.

4.3. Dissociation Energies. The dissociation energy was calculated using the above-described methodology, and the results are presented in column 4 of Table 2 . Figure 3 summarizes the results. The calculated values agree well with previously reported results. ${ }^{40}$

This graph clearly shows that the smallest anion $\left(\mathrm{BF}_{4}^{-}\right)$ always shows the highest dissociation energy, whereas for the larger anions $\mathrm{PF}_{6}^{-}$and even more so the TFSI anion, the overall negative charge is distributed over a larger number of atoms, thereby reducing the interaction with the cation and thus decreasing the dissociation energy.
Table 2. Calculated Densities $\left(\mathrm{g}^{\cdot} \mathrm{cm}^{-3}\right)$, Vaporization

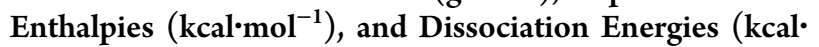
moll $^{-1}$ ) of the 18 Investigated Ionic Liquids ${ }^{a}$

\begin{tabular}{lcccc} 
ionic liquid & density & $\Delta H_{\text {vap }}$ & \multicolumn{2}{c}{$E_{\text {diss }}$ (standard deviation) } \\
$\mathrm{BMIM} / \mathrm{BF}_{4}$ & $1.170(4)$ & 32.4 & 91.83 & $(0.77)$ \\
$\mathrm{BMIM} / \mathrm{PF}_{6}$ & $1.327(5)$ & 43.2 & 80.7 & $(1.66)$ \\
$\mathrm{BMIM} / \mathrm{TFSI}$ & $1.494(4)$ & 34.4 & 76.38 & $(3.25)$ \\
$\mathrm{EMIM} / \mathrm{BF}_{4}$ & $1.245(4)$ & 36.4 & 92.95 & $(0.55)$ \\
$\mathrm{EMIM} / \mathrm{PF}_{6}$ & $1.424(5)$ & 37.7 & 82.4 & $(1.17)$ \\
$\mathrm{EMIM} / \mathrm{TFSI}$ & $1.587(4)$ & 30.7 & 77.57 & $(2.07)$ \\
$\mathrm{PIP}_{13} / \mathrm{BF}_{4}$ & $1.124(3)$ & 37.3 & 98.12 & $(1.11)$ \\
$\mathrm{PIP}_{13} / \mathrm{PF}_{6}$ & $1.278(3)$ & 28.3 & 86.59 & $(2.35)$ \\
$\mathrm{PIP}_{13} / \mathrm{TFSI}_{\mathrm{PYR}} / \mathrm{BF}_{4}$ & $1.452(4)$ & 45.6 & 79.98 & $(1.47)$ \\
$\mathrm{PYR}_{14} / \mathrm{PF}_{6}$ & $1.109(3)$ & 46.9 & 90.95 & $(0.58)$ \\
$\mathrm{PYR}$ & $1.259(3)$ & 49.0 & 80.07 & $(0.77)$ \\
$\mathrm{SBP} / \mathrm{BF}_{4}$ & $1.438(3)$ & 47.6 & 75.89 & $(1.35)$ \\
$\mathrm{SBP} / \mathrm{PF}_{6}$ & $1.199(4)$ & 44.2 & 92.14 & $(0.01)$ \\
$\mathrm{SBP} / \mathrm{TFSI}_{\mathrm{TEA}} / \mathrm{BF}_{4}$ & $1.365(4)$ & 44.2 & 81.32 & $(0.48)$ \\
$\mathrm{TEA} / \mathrm{PF}_{6}$ & $1.535(4)$ & 43.3 & 77.46 & $(0.24)$ \\
$\mathrm{TEA} / \mathrm{TFSI}$ & $1.087(3)$ & 45.3 & 89.22 & $(1.23)$ \\
$\mathrm{S}$ & $1.236(3)$ & 46.7 & 78.06 & $(0.07)$ \\
& $1.427(3)$ & 45.8 & 73.67 & $(0.74)$
\end{tabular}

${ }^{a}$ Standard deviations associated with values calculated are given in parentheses.

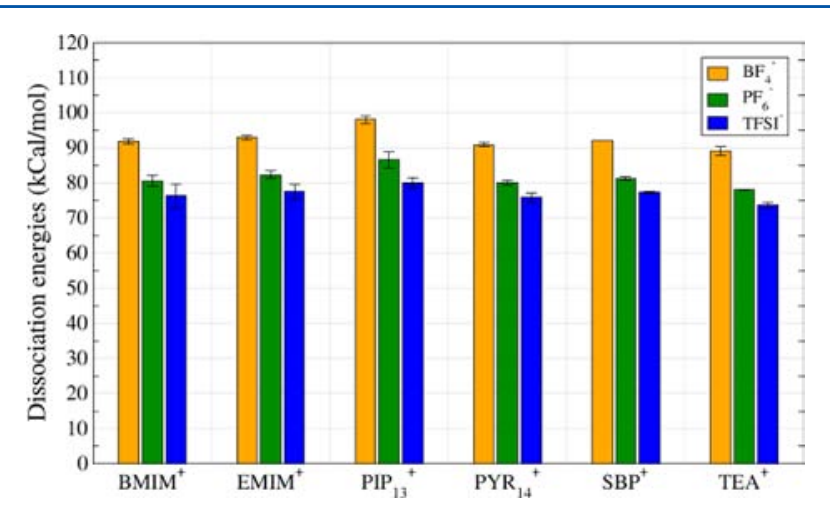

Figure 3. Dissociation energies calculated for the 18 ionic liquids.

4.4. Adsorption in Carbon Nanotubes. Figure 4 presents energy plots showing how the adsorption energy changes with the internal diameters of the carbon nanotubes for the various ions.

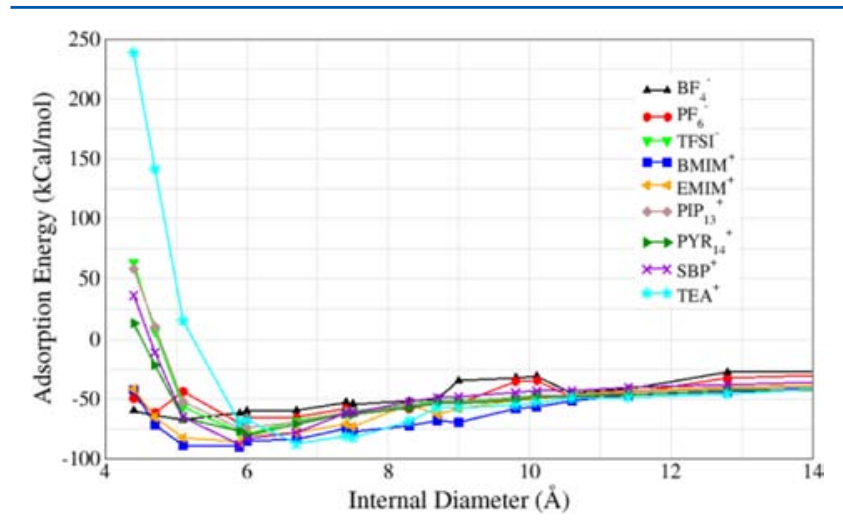

Figure 4. Adsorption energies as a function of the pore diameter of the carbon nanotube for the 18 investigated ions. 
These curves take the form of a Lennard-Jones potential: Each curve shows a minimum, and the position of the minimum depends on the size of the ion. Table 3 shows the

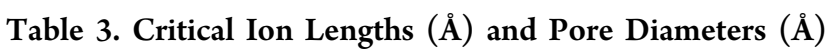
Where the Adsorption Energy Is Largest ${ }^{a}$

\begin{tabular}{lcc}
\multicolumn{1}{c}{ ion } & critical length $(\AA)$ & pore diameter $(\AA)$ \\
$\mathrm{BF}_{4}{ }^{-}$ & 5.1 & 5.1 \\
$\mathrm{PF}_{6}{ }^{-}$ & 5.4 & 5.9 \\
$\mathrm{PYR}^{-}$ & 6.4 & 6.0 \\
$\mathrm{EMIM}^{+}$ & 6.5 & 5.9 \\
$\mathrm{BMIM}^{+}$ & 6.5 & 5.9 \\
$\mathrm{TFSI}^{-}$ & 6.8 & 6.0 \\
$\mathrm{SBP}^{+}$ & 7 & 5.9 \\
$\mathrm{PIP}_{13}{ }^{+}$ & 7.1 & 5.9 \\
$\mathrm{TEA}^{+}$ & 8.1 & 6.7
\end{tabular}

${ }^{a}$ Overall charge on the electrode is $2.0 \mathrm{e}$.

second longest molecular length of each ion. This length determines whether the ion is still capable of entering the CNT (we thus assume that the greatest molecular length does not play a role, because the ion will align parallel to the axis of the $\mathrm{CNT}$ ) and can thus be considered as the critical length or diameter: If the pore diameter of the CNT becomes smaller than this "critical diameter", the ion will experience increasing repulsive forces to enter the nanotube.

From this descriptor, it follows that the "smallest" ion, $\mathrm{BF}_{4}^{-}$, has the smallest internal diameter and that the "largest" ion, $\mathrm{TEA}^{+}$, has the largest internal diameter, as is indeed is the case (Table 3). It is to be noticed that for, the ions with critical diameters of 5.4-7.1, all these ions have their energy minimum at $5.9 \AA$, because in the interval of 5.1 and $6.7 \AA$, only four nanotubes are considered to have diameters of 5.1, 5.9, 6.0, and $6.7 \AA$.

Ions exhibiting some flexibility in their geometry, for example, they might undergo changes in dihedral angles (or even bond angles), thereby increasing their internal energy only marginally, all have their optimum adsorption energies at a pore size that is slightly smaller than the critical distance. This is most pronouncedly seen for $\mathrm{TEA}^{+}$(with four flexible ethyl groups) and $\mathrm{BMIM}^{+}$(flexible $n$-butyl group). For ions that lack this flexibility, as for $\mathrm{BF}_{4}{ }^{-}$, the pore diameter for the optimum adsorption energy is very close to the critical length. In the case of $\mathrm{PF}_{6}^{-}$, it is even somewhat larger.

The adsorption energy presented in Figure 5 for each ion is the sum of intramolecular and intermolecular energies. Of course, the intermolecular energy, which is the sum of the van der Waals energy and the electrostatic energy, depends on the overall charge of the carbon nanotube. We applied a set of three overall charges: $0.5,1.0$, and 2.0 e. However, because the number of carbon atoms per cell used in the molecular simulations was not the same for all investigated CNTs, the net atomic charges on each carbon atom varied slightly (between 0.0005 and $0.0042 \mathrm{e}$ ), even though the overall charge of the CNT was the same. We are well aware that simulations with a fixed potential, instead of fixed charges, do not necessarily yield the same results, for example, for ion organization and speed of convergence. ${ }^{41}$ However, in our case, because only a single ion was simulated, there was no ion organization. Moreover, our force-field model did not allow for atomic charge polarization.

From Figure 5 it can be easily seen that the shape of the overall adsorption energy is essentially determined by the van

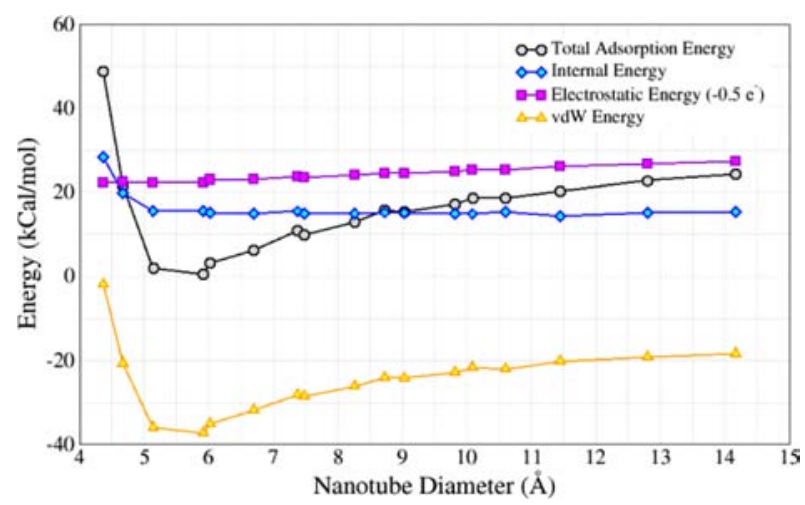

Figure 5. van der Waals contributions (yellow triangles), internal energy contributions (blue diamonds), and electrostatic energy contributions at -0.5 e (pink squares) to the total adsorption energy (black circles) of BMIM as a function of the pore diameter $(\AA)$ of the carbon nanotube.

der Waals curve. This energy contribution is attractive (negative energy value) over the whole range, but becomes positive at pore diameters smaller than $4.5 \AA$, likely because repulsive interactions dominate when the pore diameter is smaller than the critical length of $\mathrm{BMIM}^{+}(6.5 \AA)$ and the ion needs to be distorted to enter the tube. The curve shows a minimum where the ion size matches the CNT diameter.

On the other hand, the electrostatic energy and the internal energy, which is the sum of the bond, angle, and torsion energies, are both positive and thus have a destabilizing effect. The (repulsive) electrostatic energy increases slightly over the range from 4.5 to $14 \AA$. This energy term is the sum of the intramolecular electrostatic energy (independent of the CNT pore diameter) and the intermolecular (between ion and CNT) electrostatic energy, which diminishes with increasing interatomic distance (vide infra).

The internal energy becomes more positive when the pore diameter reaches the size of the critical length of the ion, as a result of a significant increase in the bond angle energy.

The impact of an increase in the overall charge of the CNT on the electrostatic energy is displayed in Figure 6.

The electrostatic energies resulting from interactions within the cation (intramolecular) always have a repulsive character and are relatively independent of the pore diameter. At low

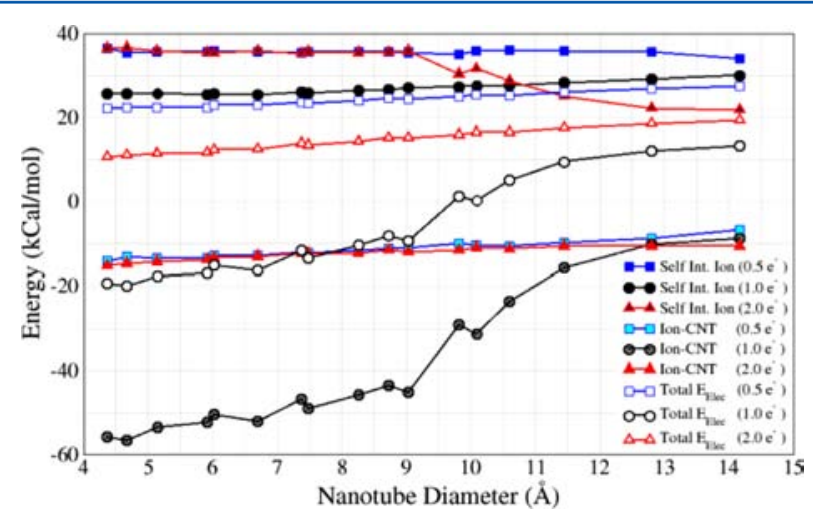

Figure 6. Contributions from the electrostatic interactions for $\mathrm{BMIM}^{+}$: intramolecular (i.e., within the ion, self), intermolecular between $\mathrm{BMIM}^{+}$and CNT, and the sum (total) of inter- and intramolecular electrostatic interactions at three different charges of the CNT: 0.5 e (squares), $1.0 \mathrm{e}$ (circles, ) and $2.0 \mathrm{e}$ (triangles). 
overall charges ( 0.5 and $1.0 \mathrm{e})$, these positive charges are not compensated by the attractive interactions between the ion and CNT. Only when the overall charge is increased to 2.0 e does the sum of the inter- and intramolecular electrostatic interactions become negative up to a diameter of $9.0 \AA$ ( $\times$ symbols). Beyond this diameter, the contribution rapidly drops to values calculated for overall charges of 0.5 and 1.0 e. Because the ion is mainly located near the surface of the CNT during the dynamics, the favorable interactions with the other $\mathrm{C}$ atoms diminish as the pore diameter increases. Eventually, they converge to a certain value when the pore diameter becomes infinite. This tendency is, of course, more pronounced as the charge on the carbon atoms increases. This is schematically depicted in Figure 7.

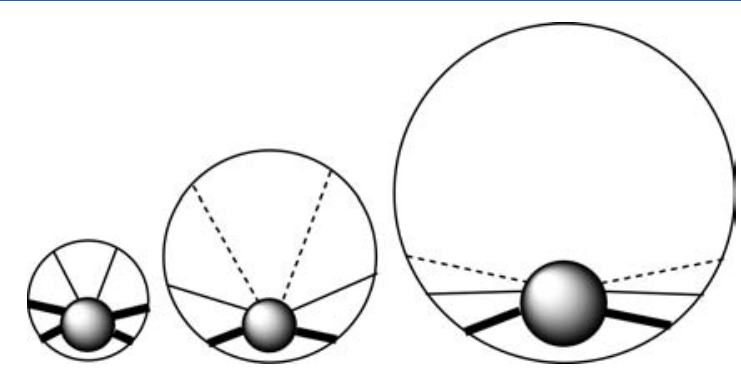

Figure 7. Two-dimensional schematic representation of the electrostatic interaction between the ion (solid circle) and CNT. As the pore diameter increases, the contributions of the $\mathrm{C}$ atoms of the CNT to $E_{\text {elec }}$ diminish (indicated by a diminishing thickness of the lines) and finally converge when the pore diameter becomes infinite.

4.5. Representing CDCs by CNTs. To better compare the calculated total energy using CNTs with the experimental capacitance for which carbide-derived carbons (CDCs) were used, we needed to correlate the two. CDCs are synthesized by the high-temperature chlorination of carbides. ${ }^{4}$ CDCs have a narrow pore size distribution with a mean value that can be tuned by varying the chlorination temperature (cf. Figure 8 ).

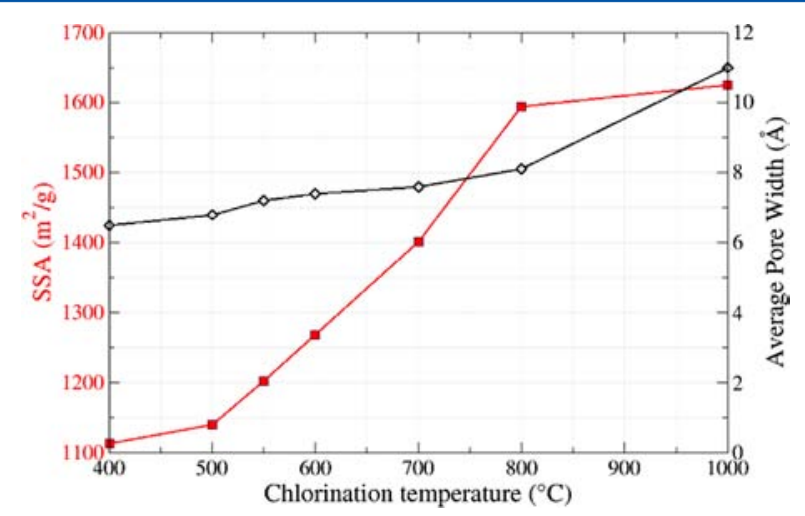

Figure 8. Effect of chlorination temperature on average pore size (diamonds) and specific surface area (squares). ${ }^{6}$

We now reasonably assume that the CDCs have a pore width distribution with a Gaussian distribution around its mean value. Instead of simulating a CDC by using a single CNT that has its diameter closest to the experimental pore width, we used a weighted distribution of several CNTs. The weights were chosen to match as closely as possible the different pore width distributions presented by Gogotsi and co-workers. ${ }^{42,43}$ It is relevant to add here that Kondrat et al. suggested that the pore size distribution is a far more important parameter than the average pore size alone, because the energy stored depends on the width of the Gaussian distribution. ${ }^{44}$ Figure 9 shows the six weighted distributions with the average pore diameters studied here.

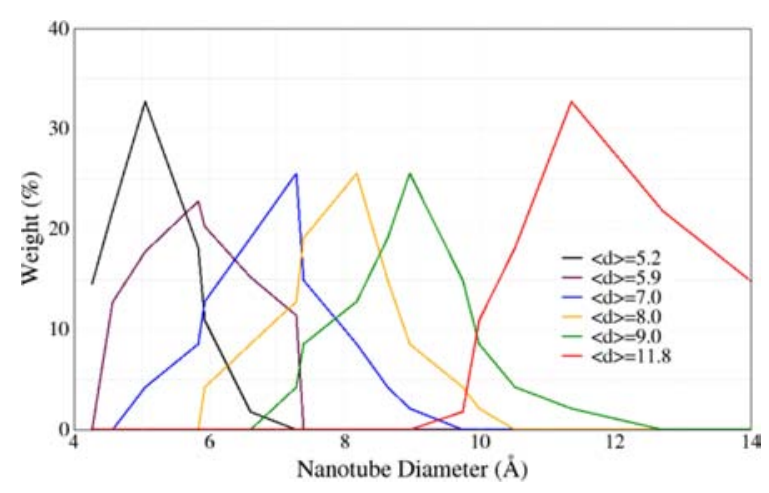

Figure 9. Pore diameter distributions for average pore diameters of 5.2, 5.9, 7.0, 8.0, 9.0, and $11.8 \AA$, using a weighted distribution of several CNTs.

We recalculated the adsorption energy for the different ions using these weighted distributions (Figure 10). It can be seen

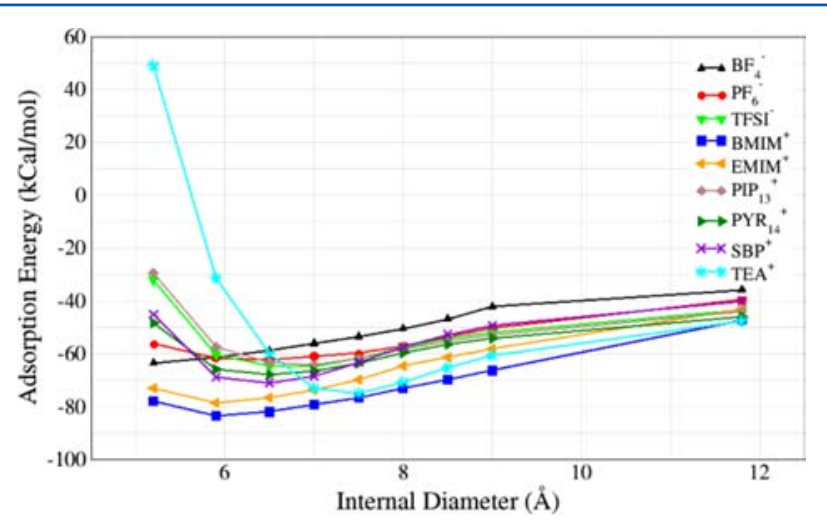

Figure 10. Adsorption energies as a function of weighted pore diameters of carbon nanotubes (with an overall charge of $2.0 \mathrm{e}$ ) for the 18 investigated ions.

that the minimum of each curve became slightly shallower, whereas its position essentially remained the same with respect to the unimodal distribution in Figure 4 and Table 4.

Table 4. Pore Diameters ( $)$ Where the Adsorption Energy Is Largest for Unimodal and Weighted Multimodal Distributions $^{a}$

\begin{tabular}{lcc}
\multicolumn{1}{c}{ ion } & unimodal & multimodal \\
$\mathrm{BF}_{4}{ }^{-}$ & 5.1 & 5.2 \\
$\mathrm{PF}_{6}^{-}$ & 5.9 & 6.5 \\
$\mathrm{PYR}_{14}{ }^{+}$ & 6.0 & 6.5 \\
$\mathrm{EMIM}^{+}$ & 5.9 & 5.9 \\
$\mathrm{BMIM}^{+}$ & 5.9 & 5.9 \\
$\mathrm{TFSI}^{-}$ & 6.0 & 7.0 \\
$\mathrm{SBP}^{+}$ & 5.9 & 6.5 \\
$\mathrm{PIP}_{13}^{+}$ & 5.9 & 7.0 \\
$\mathrm{TEA}^{+}$ & 6.7 & 7.5
\end{tabular}

${ }^{a}$ Overall charge on the electrode is 2.0 e. 
4.6. Capacitance. As previously mentioned, significant increases in capacitance using ionic liquids and electrodes with pore diameters equivalent in size to the bare ions have been reported. Figure 11 shows the capacitance for the $1.5 \mathrm{M} \mathrm{TEA} /$
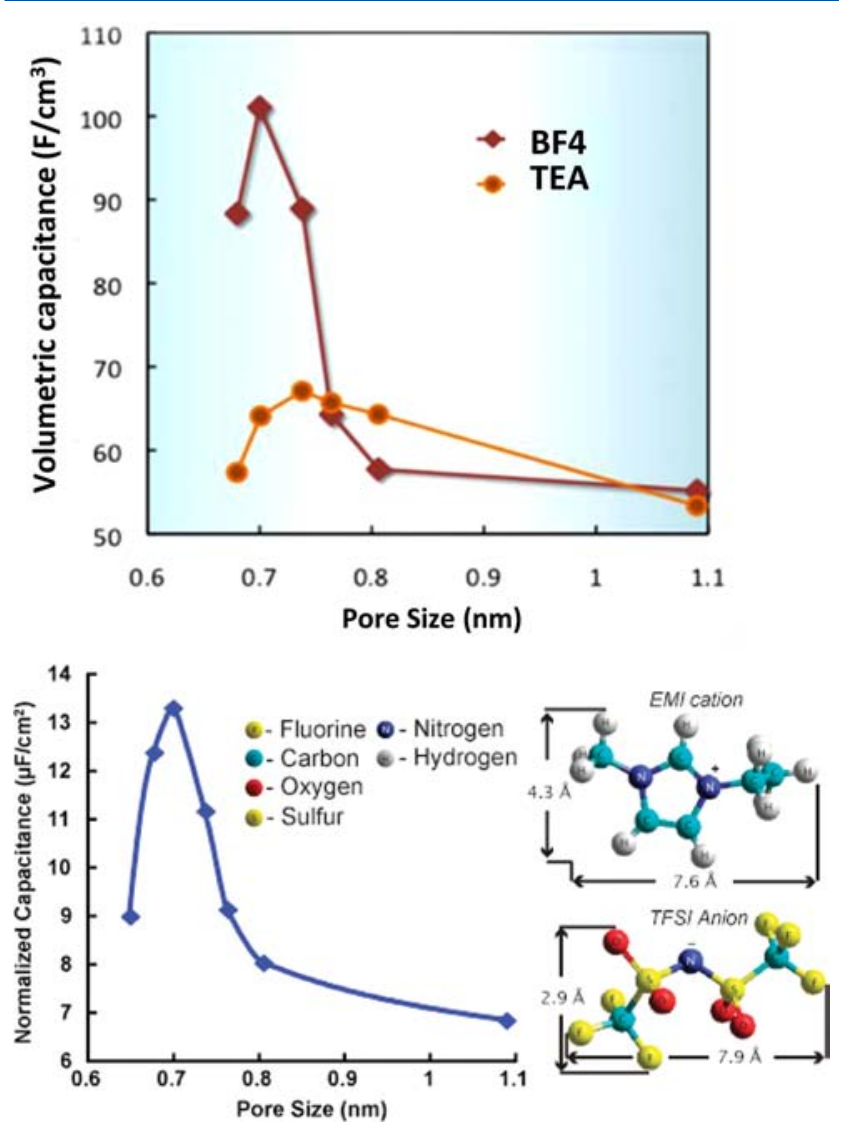

Figure 11. Measured capacitances: (top) $\mathrm{BF}_{4}^{-}$(red)/TEA ${ }^{+}$(orange), (bottom) EMI/TFSI (taken from Figure 3 of ref 6).

$\mathrm{BF}_{4}$ in acetonitrile (ACN) system (top $)^{5}$ and the EMI/TFSI system (bottom). ${ }^{6}$ It can be clearly seen that the maxima of the capacitance curve shift as a function of the dimension of the ion: The smaller $\mathrm{BF}_{4}^{-}$ion finds its maximum at a smaller pore diameter (7.0 ̊, blue curve), whereas the larger $\mathrm{TEA}^{+}$ion has a maximum at larger pore sizes $(\sim 7.5 \AA$, green curve). For the EMI/TFSI system, the (overall) maximum is found at $7.1 \AA$.

When we considered the pore diameter with maximum adsorption energy, we found very good agreement for all ions, except for the $\mathrm{BF}_{4}$ anion, for which, according to the simulations, the optimum adsorption energies were found near $5.2 \AA$. The shift to the experimentally observed maximum of $7.0 \AA$ suggests that this anion is not present as a neat anion, but is still (partially) coordinated by acetonitrile solvent molecules. This corroborates the dissociation energies calculated for this anion, which are significantly higher than those for TFSI ${ }^{-}$or $\mathrm{PF}_{6}^{-}$, clearly indicating that solvent molecules play an important role in contributing to an increase in the measured capacitance. ${ }^{5}$ We note that the maximum capacitance for $\mathrm{TEA}^{+}$ at $7.5 \AA$ corresponds well with the optimum pore diameter calculated using the multimodal model, suggesting that TEA enters the CNT as a neat ion without solvent molecules.

4.7. Ionic Liquid Screening. Because we determined the desolvation energy and dissociation energy for each ionic liquid and because we calculated the adsorption energy for each ion, as detailed in Figure 1, we can calculate the overall energy contribution for each ion as a function of the pore size of the nanoporous material. These energies are presented in Table 5, where negative (favorable) energies are indicated in bold. Overall negative energy values are obtained only if the adsorption energies outweigh the energy costs of desolvation and dissociation, that is, if the pore size is slightly greater than the dimensions of the ion and, thus, the adsorption energy becomes the most negative.

It can be seen that, if the sizes of the cation and anion are quite different, the minima of the adsorption energy are well separated, and therefore, they do not outweigh the desolvation and dissociation energies for a given nanopore diameter, so that the total energy change upon adsorption remains positive. This is seen, for example, for cations $\mathrm{PIP}_{13}{ }^{+}, \mathrm{PYR}_{14}{ }^{+}, \mathrm{SPB}^{+}$, and TEA ${ }^{+}$in combination with the $\mathrm{BF}_{4}{ }^{-}$anion. Recall that $\mathrm{BF}_{4}{ }^{-}$was

Table 5. Total Energies (kcal/mol) of the Ionic Liquids Investigated as Functions of Pore Size $(\AA)$

\begin{tabular}{|c|c|c|c|c|c|c|c|c|c|}
\hline & \multicolumn{9}{|c|}{ pore size $(\AA)$} \\
\hline & 5.2 & 5.9 & 6.5 & 7.0 & 7.5 & 8.0 & 8.5 & 9.0 & 11.8 \\
\hline $\mathrm{BMIM} / \mathrm{BF}_{4}$ & -17.0 & -20.6 & -16.5 & -11.1 & -5.7 & 0.7 & 7.7 & 15.7 & 41.1 \\
\hline $\mathrm{BMIM} / \mathrm{PF}_{6}$ & -10.1 & -21.1 & -20.1 & -16.3 & -12.3 & -6.1 & 0.7 & 7.5 & 37.0 \\
\hline BMIM/TFSI & 1.1 & -33.0 & -35.8 & -33.3 & -27.3 & -20.0 & -13.0 & -7.4 & 20.0 \\
\hline $\mathrm{EMIM} / \mathrm{BF}_{4}$ & -8.1 & -11.5 & -7.0 & -1.2 & 5.2 & 13.3 & 20.1 & 28.3 & 49.4 \\
\hline $\mathrm{EMIM} / \mathrm{PF}_{6}$ & -9.0 & -19.9 & -18.5 & -14.4 & -9.4 & -1.4 & 5.2 & 12.2 & 37.5 \\
\hline EMIM/TFSI & 3.5 & -30.5 & -32.9 & -30.0 & -23.1 & -13.9 & -7.2 & -1.4 & 21.8 \\
\hline $\mathrm{PIP}_{13} / \mathrm{BF}_{4}$ & 42.8 & 16.9 & 13.5 & 15.3 & 20.2 & 26.7 & 33.5 & 40.6 & 55.7 \\
\hline $\mathrm{PIP}_{13} / \mathrm{PF}_{6}$ & 29.5 & -3.9 & -10.4 & -10.2 & -6.7 & -0.3 & 6.2 & 12.1 & 31.4 \\
\hline $\mathrm{PIP}_{13} / \mathrm{TFSI}$ & 64.5 & 8.0 & -2.3 & -3.3 & 2.1 & 9.6 & 16.3 & 21.0 & 38.2 \\
\hline $\mathrm{PYR}_{14} / \mathrm{BF}_{4}$ & 26.1 & 10.7 & 11.2 & 15.2 & 21.0 & 27.7 & 34.7 & 41.7 & 56.2 \\
\hline $\mathrm{PYR}_{14} / \mathrm{PF}_{6}$ & 24.6 & 1.7 & -0.8 & 1.5 & 5.9 & 12.5 & 19.2 & 25.0 & 43.7 \\
\hline $\mathrm{PYR}_{14} / \mathrm{TFSI}$ & 43.3 & -2.7 & -9.0 & -7.9 & -1.6 & 6.2 & 13.0 & 17.7 & 34.3 \\
\hline $\mathrm{SBP} / \mathrm{BF}_{4}$ & 28.0 & 6.4 & 6.7 & 12.1 & 19.7 & 28.5 & 36.9 & 45.0 & 60.6 \\
\hline $\mathrm{SBP} / \mathrm{PF}_{6}$ & 24.4 & -4.7 & -7.5 & -3.8 & 2.5 & 11.2 & 19.3 & 26.2 & 46.0 \\
\hline SBP/TFSI & 44.0 & -8.2 & -14.8 & -12.4 & -4.2 & 5.7 & 13.9 & 19.7 & 37.4 \\
\hline TEA/BF ${ }_{4}$ & 120.1 & 42.0 & 15.8 & 5.6 & 6.1 & 13.4 & 22.4 & 31.8 & 51.2 \\
\hline TEA/ $\mathrm{PF}_{6}$ & 117.6 & 32.0 & 2.7 & -9.1 & -10.0 & -2.8 & 5.9 & 14.2 & 37.6 \\
\hline TEA/TFSI & 136.6 & 27.8 & -5.2 & -18.3 & -17.2 & -8.9 & -0.1 & 7.0 & 28.4 \\
\hline
\end{tabular}


simulated as a neat anion without any solvent molecules (such as $\mathrm{ACN}$ ).

Using these overall energy values, we now have a guideline for the selection of the ionic liquids with the largest capacitances for a given pore size, or vice versa. For example, the couple BMIM/TFSI would give one of the highest capacitances for a pore size around $6.5 \AA$, whereas BMIM/ $\mathrm{BF}_{4}$ is more adequate for pore sizes near $5 \AA$. Additionally, on the basis of overall energy changes, it can be reasonably expected that, for the pore distribution around $6.5 \AA$, the capacitance is highest. However, the use of asymmetric electrodes (i.e., electrodes with different average pore sizes) is recommended if ionic liquids in which the anions and cations have substantially different geometrical sizes are used.

\section{CONCLUSIONS AND PERSPECTIVES}

In this article, we have shown that the energy curves related to ion (of an ionic liquid as an electrolyte in a capacitor) adsorption from the bulk solution into carbon nanotubes with varying diameters correlates with the normalized/specific capacitance curves, as a function of the pore diameter of the carbonaceous electrode material. To this end, the desolvation of an ion pair from the bulk solution was calculated using molecular dynamics simulations, and its dissociation energy was estimated using B3LYP calculations. An original method was presented to calculate the adsorption of the ion in the nanopores of the electrode (represented by carbon nanotubes) in which the total charge of the ion is not necessarily compensated by the overall charge of the CNT of the electrode.

Favorable adsorption energies outweigh the desolvation and dissociation energies only if the pore size of the nanopore is slightly greater than the size of the ion, that is, the ion is well enveloped by the carbon atoms of the nanotube and the favorable van der Waals interaction become optimal. If the pore size gets smaller than the ion, the ion needs to be deformed to enter the pore, and this severely penalizes the adsorption energy. On the other hand, if the pore size is significantly larger than the ion, favorable van der Waals interactions are too small to compensate for the dissociation and desolvation energies, and the total energy balance remains negative.

If one considers symmetric electrodes, the most favorable adsorptions energies are obtained when both anions and cations have comparable dimensions. $\mathrm{TSFI}^{-}$is comparable in size to the investigated cations and larger than $\mathrm{BF}_{4}^{-}$and $\mathrm{PF}_{6}^{-}$. Moreover, larger anions would have smaller dissociation energies as compared to $\mathrm{BF}_{4}^{-}$, positively impacting the overall energy change, $\Delta E_{\text {tot }}$ in Figure 1.

\section{AUTHOR INFORMATION}

\section{Corresponding Author}

*E-mail: theodorus.de-bruin@ifpen.fr.

\section{Author Contributions}

The manuscript was written through contributions of all authors.

\section{Notes}

The authors declare no competing financial interest.

\section{ACKNOWLEDGMENTS}

We are grateful for the computing resources on JADE (CINES) and Titane (CCRT) of the French National HPC obtained through Project x2012096349. We acknowledge the support of the French Agence Nationale de la Recherche (ANR) under
Grant ANR-2010-0933-02 ("Modeling Ion Adsorption in Carbon NANOpores").

\section{REFERENCES}

(1) Simon, P.; Gogotsi, Y. Materials for Electrochemical Capacitors. Nat. Mater. 2008, 7, 845-854.

(2) Gamby, J.; Taberna, P. L.; Simon, P.; Fauvarque, J. F.; Chesneau, M. Studies and Characterisations of Various Activated Carbons Used for Carbon/Carbon Supercapacitors. J. Power Sources 2001, 101, 109116.

(3) Mi, J.; Wang, X.-R.; Fan, R.-J.; Qu, W.-H.; Li, W.-C. CoconutShell-Based Porous Carbons with a Tunable Micro/Mesopore Ratio for High-Performance Supercapacitors. Energy Fuels 2012, 26, 53215329.

(4) Chmiola, J.; Yushin, G.; Gogotsi, Y.; Portet, C.; Simon, P.; Taberna, P.-L. Anomalous Increase in Carbon Capacitance at Pore Sizes Less Than 1 Nanometer. Science 2006, 313, 1760-1763.

(5) Chmiola, J.; Largeot, C.; Taberna, P.-L.; Simon, P.; Gogotsi, Y. Desolvation of Ions in Subnanometer Pores and Its Effect on Capacitance and Double-Layer Theory. Angew. Chem., Int. Ed. 2008, 47, 3392-3395.

(6) Largeot, C.; Portet, C.; Chmiola, J.; Taberna, P.-L.; Gogotsi, Y.; Simon, P. Relation Between the Ion Size and Pore Size for an Electric Double-Layer Capacitor. J. Am. Chem. Soc. 2008, 130, 2730-2731.

(7) Segalini, J.; Iwama, E.; Taberna, P.-L.; Gogotsi, Y.; Simon, P. Steric Effects in Adsorption of Ions from Mixed Electrolytes into Microporous Carbon. Electrochem. Commun. 2012, 15, 63-65.

(8) Huang, J.; Sumpter, B. G.; Meunier, V. Theoretical Model for Nanoporous Carbon Supercapacitors. Angew. Chem., Int. Ed. 2008, 47, 520-524.

(9) Feng, G.; Li, S.; Presser, V.; Cummings, P. T. Molecular Insights into Carbon Supercapacitors Based on Room Temperature Ionic Liquids. J. Phys. Chem. Lett. 2013, 4, 3367-3376.

(10) Shim, Y.; Kim, H. J. Nanoporous Carbon Supercapacitors in an Ionic Liquid: A Computer Simulation Study. ACS Nano 2010, 4, 2345-2355.

(11) Yang, L.; Fishbine, B. H.; Migliori, A.; Pratt, L. R. Molecular Simulation of Electric Double-Layer Capacitors Based on Carbon Nanotube Forests. J. Am. Chem. Soc. 2009, 131, 12373-12376.

(12) Merlet, C.; Rotenberg, B.; Madden, P. A.; Taberna, P.-L.; Simon, P.; Gogotsi, Y.; Salanne, M. On the Molecular Origin of Supercapacitance in Nanoporous Carbon Electrodes. Nat. Mater. 2012, 11, 306-310.

(13) Canongia Lopes, J. N.; Deschamps, J.; Padua, A. A. H. Modeling Ionic Liquids Using a Systematic All-Atom Force Field. J. Phys. Chem. B 2004, 108, 11250-11250.

(14) Canongia Lopes, J. N.; Padua, A. A. H. Molecular Force Field for Ionic Liquids III: Imidazolium, Pyridinium, and Phosphonium Cations; Chloride, Bromide, and Dicyanamide Anions. J. Phys. Chem. B 2006, 110, 19586-19592.

(15) Andrade, J.; de Böes, E. S.; Stassen, H. A Force Field for Liquid State Simulations on Room Temperature Molten Salts: 1-Ethyl-3methylimidazolium Tetrachloroaluminate. J. Phys. Chem. B 2002, 106, 3546-3548.

(16) Kaminski, G. A.; Jorgensen, W. L. Host-Guest Chemistry of Rotaxanes and Catenanes: Application of a Polarizable All-Atom Force Field to Cyclobis(paraquat-p-phenylene) Complexes with Disubstituted Benzenes and Biphenyls. J. Chem. Soc., Perkin Trans. 2 1999, 2365-2375.

(17) Canongia Lopes, J. N. Molecular Force Field for Ionic Liquids Composed of Triflate or Bistriflylimide Anions. J. Phys. Chem. B 2004, 16893-16898.

(18) Smith, W. Molecular Simulation with DL POLY Classic. http://www.ccp5.ac.uk/DL_POLY_CLASSIC/ (accessed April 2013).

(19) Martínez, L.; Andrade, R.; Birgin, E. G.; Martínez, J. M. PACKMOL: A Package for Building Initial Configurations for Molecular Dynamics Simulations. J. Comput. Chem. 2009, 30, 215764. 
(20) Allen, M. P.; Tildesley, D. J. Computer Simulation of Liquids; Oxford University Press: Oxford, U.K., 1987.

(21) Roy, D.; Patel, N.; Conte, S.; Maroncelli, M. Dynamics in an Idealized Ionic Liquid Model. J. Phys. Chem. B 2010, 114, 8410-8424.

(22) Roy, D.; Maroncelli, M. An Improved Four-Site Ionic Liquid Model. J. Phys. Chem. B 2010, 114, 12629-12631.

(23) Hanke, C. G.; Price, S. L.; Lynden-Bell, R. M. Intermolecular Potentials for Simulations of Liquid Imidazolium Salts. Mol. Phys. 2001, 99, 801-809.

(24) Shah, J. K.; Brennecke, J. F.; Maginn, E. J. Thermodynamic Properties of the Ionic Liquid 1-n-Butyl-3-methylimidazolium Hexafluorophosphate from Monte Carlo Simulations. Green Chem. 2002, 4, 112-118

(25) Shah, J. K.; Maginn, E. J. A Monte Carlo Simulation Study of the Ionic Liquid 1-n-Butyl-3-methylimidazolium Hexafluorophosphate: Liquid Structure, Volumetric Properties and Infinite Dilution Solution Thermodynamics of $\mathrm{CO}_{2}$. Fluid Phase Equilib. 2004, 222-223, 195203.

(26) Borodin, O.; Smith, G. D.; Henderson, W. $\mathrm{Li}^{+}$Cation Environment, Transport, and Mechanical Properties of the LiTFSI Doped $\mathrm{N}$-Methyl- $\mathrm{N}$-alkylpyrrolidinium ${ }^{+} \mathrm{TFSI}^{-}$Ionic Liquids. J. Phys. Chem. B 2006, 110, 16879-16886.

(27) Borodin, O.; Smith, G. D.; Fan, P. Molecular Dynamics Simulations of Lithium Alkyl Carbonates. J. Phys. Chem. B 2006, 110, 22773-22779.

(28) Borodin, O.; Smith, G. D. Structure and Dynamics of N-Methyl$\mathrm{N}$-propylpyrrolidinium Bis(trifluoromethanesulfonyl)imide Ionic Liquid from Molecular Dynamics Simulations. J. Phys. Chem. B 2006, 110, 11481-11490.

(29) Borodin, O. Polarizable Force Field Development and Molecular Dynamics Simulations of Ionic Liquids. J. Phys. Chem. B 2009, 113, 11463-11478

(30) Smith, G. D.; Borodin, O.; Russo, S. P.; Rees, R. J.; Hollenkamp, A. F. A Molecular Dynamics Simulation Study of $\mathrm{LiFePO}_{4} /$ Electrolyte Interfaces: Structure and $\mathrm{Li}^{+}$Transport in Carbonate and Ionic Liquid Electrolytes. Phys. Chem. Chem. Phys. 2009, 11, 9884-9897.

(31) Bedrov, D.; Borodin, O.; Li, Z.; Smith, G. D. Influence of Polarization on Structural, Thermodynamic, and Dynamic Properties of Ionic Liquids Obtained from Molecular Dynamics Simulations. J. Phys. Chem. B 2010, 114, 4984-4997.

(32) Borodin, O.; Gorecki, W.; Smith, G. D.; Armand, M. Molecular Dynamics Simulation and Pulsed-Field Gradient NMR Studies of Bis(fluorosulfonyl)imide (FSI) and Bis[(trifluoromethyl)sulfonyl]imide (TFSI)-Based Ionic Liquids. J. Phys. Chem. B 2010, 114, 6786-6798.

(33) Vatamanu, J.; Borodin, O.; Smith, G. D. Molecular Insights into the Potential and Temperature Dependences of the Differential Capacitance of a Room-Temperature Ionic Liquid at Graphite Electrodes. J. Am. Chem. Soc. 2010, 132, 14825-14833.

(34) Smith, G. D.; Borodin, O.; Magda, J. J.; Boyd, R. H.; Wang, Y.; Bara, J. E.; Miller, S.; Gin, D. L.; Noble, R. D. A Comparison of Fluoroalkyl-Derivatized Imidazolium:TFSI and Alkyl-Derivatized Imidazolium:TFSI Ionic Liquids: A Molecular Dynamics Simulation Study. Phys. Chem. Chem. Phys. 2010, 12, 7064-7076.

(35) Vatamanu, J.; Cao, L. L.; Borodin, O.; Bedrov, D.; Smith, G. D. On the Influence of Surface Topography on the Electric Double Layer Structure and Differential Capacitance of Graphite/Ionic Liquid Interfaces. J. Phys. Chem. Lett. 2011, 2, 2267-2272.

(36) Vatamanu, J.; Borodin, O.; Smith, G. D. Molecular Simulations of the Electric Double Layer Structure, Differential Capacitance, and Charging Kinetics for $\mathrm{N}$-Methyl- $\mathrm{N}$-propylpyrrolidinium Bis(fluorosulfonyl)imide at Graphite Electrodes. J. Phys. Chem. B 2011, $115,3073-3084$.

(37) Sambasivarao, S. V; Acevedo, O. Development of OPLS-AA Force Field Parameters for 68 Unique Ionic Liquids. J. Chem. Theory Comput. 2009, 5, 1038-1050.

(38) Rajput, N. N.; Monk, J.; Singh, R.; Hung, F. R. On the Influence of Pore Size and Pore Loading on Structural and Dynamical
Heterogeneities of an Ionic Liquid Confined in a Slit Nanopore. J. Phys. Chem. C 2012, 116, 5169-5181.

(39) Jaguar, version 7.9; Schrödinger, LLC: New York, 2012.

(40) Fernandes, A. M.; Rocha, M. A. A.; Freire, M. G.; Marrucho, I. M.; Coutinho, A. P.; Santos, L. M. N. B. F. Evaluation of CationAnion Interaction Strength in Ionic Liquids. J. Phys. Chem. B 2011, 115, 4033-4041.

(41) Merlet, C.; Péan, C.; Rotenberg, B.; Madden, P. A.; Simon, P.; Salanne, M. Simulating Supercapacitors: Can We Model Electrodes As Constant Charge Surfaces? J. Phys. Chem. Lett. 2013, 4, 264-268.

(42) Presser, V.; Heon, M.; Gogotsi, Y. Carbide-Derived CarbonsFrom Porous Networks to Nanotubes and Graphene. Adv. Funct. Mater. 2011, 21, 810-833.

(43) Gogotsi, Y.; Nikitin, A.; Ye, H.; Zhou, W.; Fischer, J. E.; Yi, B.; Foley, H. C.; Barsoum, M. W. Nanoporous Carbide-Derived Carbon with Tunable Pore Size. Nat. Mater. 2003, 2, 591-4.

(44) Kondrat, S.; Pérez, C. R.; Presser, V.; Gogotsi, Y.; Kornyshev, A. A. Effect of Pore Size and Its Dispersity on the Energy Storage in Nanoporous Supercapacitors. Energy Environ. Sci. 2012, 5, 6474-6479. 